\title{
Small Scale Yielding Near a Crack in Plane Strain: A Finite Element Analysis
}

\author{
N. LEVY, P. V. MAR CA L, W. J. OSTERGREN^, AND J. R. RICE \\ Division of Engineering, Brown University, Providence, R. I.
}

(Received January 20, 1970)

\begin{abstract}
This paper presents an incremental elastic-plastic finite element solution to the problem of small scale yieldıng near a crack in plane strain for a non-hardening material. Emphasis is placed on the design of finite elements which allow accurate reproduction, in a numerical solution, of the detailed structure of the near crack tip stress and strain fields, as understood from previous analytical studies of crack tip singularities. Numerical results are presented for: location of the elastic-plastic boundary, angular distnbution of the strength of the strain singularity, crack tip opening displacement, stress distribution ahead of the crack, and angular variation of stresses at the tip. The latter results verify Rice's prediction of the Prandtl field in providing the limiting stress distribution as $r \rightarrow 0$.
\end{abstract}

\section{Introduction}

Recent studies by Cherepanov [1], Hutchinson [2,3], Rice [4, 5], and Rice and Rosengren [6] bave elucidated the structure of singularities at crack tips in elastic-plastic materials, particularly for the plane strain case. Our aim in this paper is to show how these studies naturally suggest a design of finite elements which allows accurate reproduction, in numerical solutions, of the principal features of the near crack tip stress and strain fields. We are, of course, addressing the problem of attaining numerical accuracy near singularities and pointing out that chances are very much enhanced if one knows (as is now the case for cracks) the functional form of the singularity. Rice and Johnson [7] have recently emphasized the importance of accurate determinations of the stress and deformation state very near the crack tip, in connecting continuum analyses with microstructural separation mechanisms so as to predict fracture toughness.

We illustrate the method through the incremental elastic-plastic solution of a problem which is of basic importance for fracture mechanics : small scale contained plastic yielding near a crack under plane strain conditions. The solution is given for the case of a non-hardening Mises material, and Rice's $[4,5]$ boundary layer formulation of the problem is adopted with the Irwin-Williams [8] inverse square root elastic singularity setting asymptotic boundary conditions.

Many numerical elastic-plastic solutions for cracked or sharply notched bodies have been published, employing finite element and finite difference methods. Representative of these are the works of Marcal and King [9], Mendelson [10], Swedlow and co-workers [11, 12], and Tuba [13]. Their work serves to elucidate general features of the growth of the yielded zone at a notch tip but, for reasons discussed subsequently, does not provide an accurate description of the stress and strain distribution very near the tip singularity where fracturing is imminent. An exception is in the numerical approach of Hilton and Hutchinson [14] who, in presenting a deformation plasticity solution to a plane stress crack problem, employed an analytical representation [2] of the dominant singularity inside a small circular region very near the tip, with finite elements employed outside.

«Presently with Generator Department, General Electric Company, Schenectady, New York. 


\section{Near Crack Tip Singularities in Plane Strain}

For this discussion, let $r, \theta, z$ be a cylindrical polar system so that the $z$ axis is perpendicular to the plane of straining and coincident with the crack tip, and that $\theta= \pm \pi$ describes the crack surfaces. Results to be summarized are based on the conventional infinitesimal displacement gradient approximation and assume plane strain conditions as induced by loadings acting symmetrically relative to the crack line. Linear elastic solutions for isotropic and homogeneous materials then lead to the characteristic Irwin-Williams singularity in stress components:

$$
\begin{aligned}
& \sigma_{\theta \theta}+\sigma_{r r}=\sigma_{r x} / \nu \rightarrow 2 K(2 \pi r)^{-\frac{1}{2}} \cos \theta / 2 \\
& \sigma_{\theta \theta}-\sigma_{r r}+2 i \sigma_{r \theta} \rightarrow i K(2 \pi r)^{-\frac{1}{2}} \sin \theta \exp i \theta / 2 .
\end{aligned}
$$

Here $i$ is the unit imaginary number, and $K$ is Irwin's stress intensity factor. $K=\sigma(\pi l / 2)^{\frac{1}{2}}$ for the Inglis configuration of a crack of length $l$ in an infinite body under the remote tensile stress $\sigma$. Values for many other configurations are given in Ref. [15].

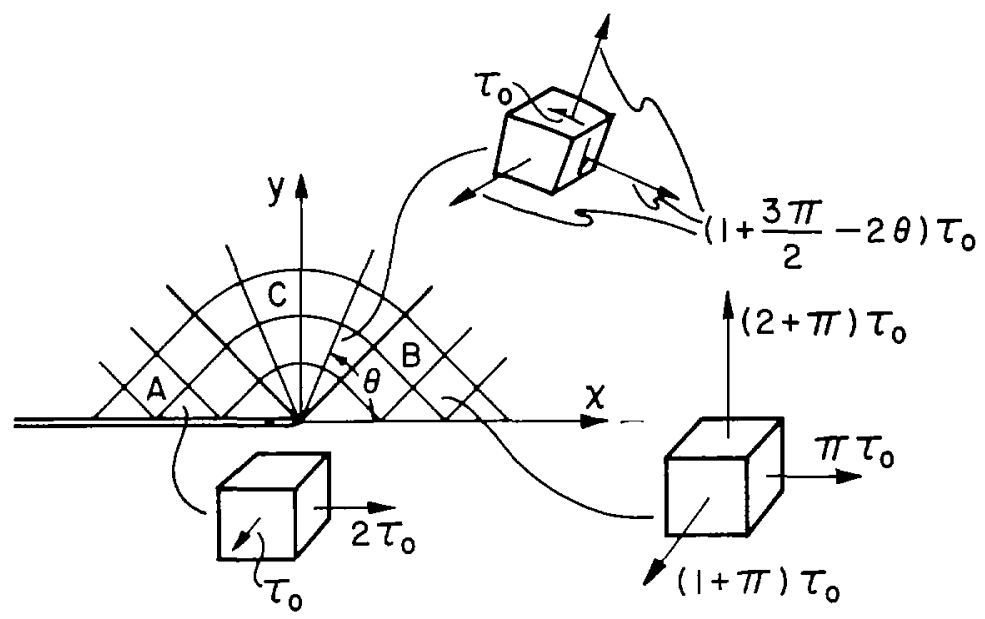

Figure 1. Prandtl slip line field representing limiting stress state as $r \rightarrow 0$ at the crack tip, from Rice [4].

In the case of an isotropic non-hardening elastic-plastic material with yield stress $\tau_{0}$ in simple shear, Rice [4] noted the role of the Prandtl slip line field and associated stress distribution, as illustrated in Fig. 1, in providing the limiting stress state as $r \rightarrow 0$ for cases of contained plastic yielding near a crack tip. The displacement components associated with this slip line pattern vary with angle $\theta$ as the crack tip is approached through the centered fans above and below the tip. Thus, there is no unique set of displacements at the tip, a different set resulting as the tip is approached along each different radial line. Writing $u_{k}(r, \theta)$ for displacements, one has in the fan (region C of Fig. 1, $\pi / 4<\theta<3 \pi / 4$ )

$$
u_{\theta}(0, \theta)=-f(\theta), \quad u_{r}(0, \theta)=f^{\prime}(\theta) .
$$

On the other hand, as the tip is approached through the constant stress regions $A$ and $B$, there is a unique set of cartesian displacement components for all $\theta$. No strain singularities result in $A$ and $B$, but as the tip is approached in $C$, the shear strain $\varepsilon_{r \theta}$ becomes infinite as

$$
\varepsilon_{r \theta} \rightarrow \frac{f^{\prime \prime}(\theta)+f(\theta)}{2 r}
$$

while $\varepsilon_{\theta \theta}$ and $\varepsilon_{r}$ remain finite. Following Ref. [4], we define a function $R(\theta)=E\left[f^{\prime \prime}(\theta)+\right.$ $f(\theta)] / 2 \tau_{0}(1+v)$, so that Eq. (3) becomes

$$
\varepsilon_{r \theta} \rightarrow \frac{(1+v) \tau_{0}}{E} \frac{R(\theta)}{r}
$$


The factor multiplying $R(\theta) / r$ is the initial yield strain in pure shear, and hence we may interpret $R(\theta)$ as an approximate measure of the extent of the plastically deformed region. The crack tip opening displacement $\delta_{t}$ (total separation distance between upper and lower crack surfaces at $r=0$ ) may be expressed as [4]

$$
\delta_{\mathrm{t}}=\frac{4(1+v) \tau_{0}}{E} \int_{\pi / 4}^{3 \pi / 4} R(\theta) \sin \theta \mathrm{d} \theta .
$$

In formulating fracture criteria, it is essential to understand the finite geometry change effects occurring adjacent to the blunting crack tip, for comparison of metallurgical size scales with values of the opening displacement at fracture reveals that processes such as ductile void growth from inclusions and cleavage micro-cracking occur in the region where large geometry change effects dominate $[7,16]$. Rice and Johnson [7] show, however, that the solution based on the small geometry change assumption can be employed to set boundary conditions on the blunting analysis. For their method, it is essential that the displacement components (2) predicted in the former solution be known accurately at the crack tip, and this is therefore a requirement to be met in useful numerical formulations of solution methods. While no exact solution is now available for the displacements (or equivalently, $R(\theta)$ ), Rice's $J$ integral technique [4] can be employed if one adopts the approximation of a deformation rather than incremental plasticity theory. In this case,path independence of the $J$ integral is assured, and by evaluating the integral on a circuit of zero radius about the tip, one has

$$
J=\frac{4(1+v) \tau_{0}^{2}}{E} \int_{\pi / 4}^{3 \pi / 4} R(\theta)[\cos \theta+(1+3 \pi / 2-2 \theta) \sin \theta] \mathrm{d} \theta .
$$

On the other hand, if one considers small scale yielding with approach in the far field to the characteristic elastic singularity (1), the integral may be directly evaluated as

$$
J=\frac{1-v^{2}}{E} K^{2}
$$

This allows some crude approximations to be made for $R(\theta)$, one [4] of which will be discussed subsequently in comparison with numerical results.

For strain hardening materials exhibiting a power law relation in the plastic range between stress and strain in simple shear ( $\tau$ proportional to $\varepsilon^{N}$, where $N$ is the hardening exponent), Cherepanov [1], Hutchinson [2], and Rice and Rosengren [6] have shown that singular near tip fields of the form

$$
\sigma_{i j} \rightarrow\left(\frac{L}{r}\right)^{N / 1+N} \Sigma_{i j}(\theta), \quad \varepsilon_{i j} \rightarrow\left(\frac{L}{r}\right)^{1 / 1+N} E_{i j}(\theta)
$$

result. The length parameter $L$ is undetermined by their analysis, but within the approximation of a deformation theory it may be directly related to the value of the $J$ integral and hence (Eq. 7) to the elastic stress intensity factor in the case of small scale yielding. Extensive result are presented in Ref. [6] for the plane strain case, with various hardening exponents. When $N=0$ (perfect plasticity) $\Sigma_{\imath j}(\theta)$ describes the Prandtl stress field of Fig. 1 . The associated angular distribution of strain $E_{i j}(\theta)$ is non-unique in that case, but we shall compare subsequent numerical results with the predicted strain distribution of the hardening solution in the limit $N \rightarrow 0^{+}$.

With these results on crack tip singularities as preliminaries, let us now turn to the formulation of a finite element numerical solution method for elastic-plastic crack problems.

\section{Finite Elements}

We have employed a general purpose elastic-plastic finite element program in this study, as developed at Brown University by Marcal and co-workers, based on the method of Marcal and King [9]. The program is open-ended in the sense that any constitutive law and any element 
design can be employed. In outlining the approach here, however, we shall limit attention to an elastically isotropic non-hardening Mises material, and concentrate on the design of finite elements which may allow numerical solutions to reproduce the main features of near tip singularities.

The incremental stress-strain relations are

$$
\frac{1+v}{E} \dot{\sigma}_{i j}=\dot{\varepsilon}_{i j}+\frac{v}{1-2 v} \delta_{i j} \dot{\varepsilon}_{k k}-\phi s_{i j} \frac{3 s_{k l} \dot{\varepsilon}_{k l}}{2 \sigma_{0}^{2}}
$$

where $s_{i j}$ is the stress deviator, $\sigma_{0}\left(=\sqrt{3} \tau_{0}\right)$ is the tensile yield stress, and where

$$
\begin{array}{ll}
\phi=0 \quad \text { if } 3 s_{i j} s_{i j}<2 \sigma_{0}^{2} \text { or if } s_{i j} \dot{\varepsilon}_{i j}<0, \\
\phi=1 \text { otherwise. }
\end{array}
$$

A proper solution for surface traction rates $T_{i}$ on the boundary curve $\Gamma$ of a planar region $A$ is given by minimizing

$$
I=\int_{A} \frac{1}{2} \dot{\sigma}_{i j}(\dot{\varepsilon}) \dot{\varepsilon}_{i j} \mathrm{~d} A-\int_{\Gamma} T_{i} \dot{u}_{i} \mathrm{~d} s
$$

on the class of all compatible strain rate-displacement rate fields [17]. Following Zienkiewicz [18], the finite element equations are derived by dividing the region $A$ into a set of finite elements within which displacement components are assumed to vary with position in such a way that (a) the displacements of any point within an element are determined by the displacements of certain "nodal" points on the boundary of that element, and (b) continuity of displacement from element to element is assured for arbitrary choices of the nodal displacements. $I$ then may be thought of as a function of the nodal displacement rates $\dot{u}_{i}^{P}$, where $P=1,2,3, \ldots$ denotes the nodes:

$$
I=\frac{1}{2} C_{i j}^{P Q} \dot{u}_{i}^{P} \dot{u}_{j}^{Q}-f_{i}^{Q} \dot{u}_{t}^{Q},
$$

so that the governing discretized equations are

$$
C_{i j}^{P Q} \dot{u}_{j}^{Q}=\dot{f}_{l}^{P} \text {. }
$$

Here $\dot{f}_{i}^{P}$ are the prescribed nodal forces, and $C_{i j}^{P Q}$ is the incremental master stiffness matrix. It is symmetric, depends on the current state of stress within elements in the plastic range, and also on the direction of the nodal displacement rates on plastic elements (loading vs. unloading).

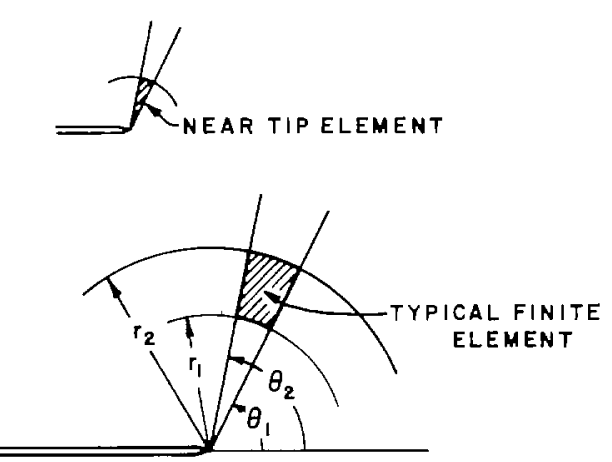

Figure 2. Typical finite element, bounded by rays and circles of a polar coordinate system with origin at the crack tip.

Marcal and King [9] have discussed techniques of assembling the stiffness matrix and iterative solution methods for the elastic-plastic range, which are adopted in our present work. Of course, the rates in (13) are replaced by small finite increments in each step of the numerical solution.

A finite element design, capable of reproducing the main features of the crack tip singularity in the plastic range, is shown in Fig. 2 . Concentric circles $(r=$ const) and rays $(\theta=$ const) form 
the element boundaries. A displacement component $u_{t}(r, \theta)(i=r, \theta)$ within an element is assumed in terms of nodal displacements as

$$
\begin{aligned}
u_{i}(r, \theta)=\frac{1}{\left(r_{2}-r_{1}\right)\left(\theta_{2}-\theta_{1}\right)}[ & {\left[\left(r_{2}-r\right)\left(\theta_{2}-\theta\right) u_{i}\left(r_{1}, \theta_{1}\right)+\left(r-r_{1}\right)\left(\theta_{2}-\theta\right) u_{i}\left(r_{2}, \theta_{1}\right)\right.} \\
& \left.+\left(r_{2}-r\right)\left(\theta-\theta_{1}\right) u_{i}\left(r_{1}, \theta_{2}\right)+\left(r-r_{1}\right)\left(\theta-\theta_{1}\right) u_{i}\left(r_{2}, \theta_{2}\right)\right] .
\end{aligned}
$$

This guarantees displacement continuity from element to element. When $r_{1}$ is zero, the two nodal points $\left(r_{1}, \theta_{1}\right)$ and $\left(r_{1}, \theta_{2}\right)$ coincide, resulting in an element nearest the crack tip such as that shown in the insert of Fig. 2. The nodal points are, however, still considered as separate and able to displace independently. Thus the elements permit the singular crack tip displacement variation anticipated in the last section. It is clear that the crack tip is the coincident site of as many independent nodal points as there are rays emanating from the crack tip. Within the angular range of a particular tip element, Eq. (14) leads to the displacement variation

$$
u_{i}(0, \theta)=\frac{1}{\left(\theta_{2}-\theta_{1}\right)}\left[\left(\theta_{2}-\theta\right) u_{i}\left(0, \theta_{1}\right)+\left(\theta-\theta_{1}\right) u_{i}\left(0, \theta_{2}\right)\right]
$$

at $r=0$. Further it is straightforward to see that the polar coordinate strain components $\varepsilon_{r r}, \varepsilon_{r \theta}, \varepsilon_{\theta \theta}$ within each element take the form

$$
\varepsilon_{i j}(r, \theta)=\frac{A_{i j}(\theta)}{r}+B_{i j}(\theta),
$$

where $A_{i j}$ and $B_{1 j}$ are linear functions of $\theta$ and the nodal displacements. Thus the elements allow duplication in the numerical solution of the $1 / r$ strain singularity anticipated from studies summarized earlier on the non-hardening elastic-plastic near tip field. Of course, the coincident nodal points at the crack tip may alternately move as a single point and thus describe a rigid body displacement as in the elastic case or in the constant state regions ( $A$ and $B$ of Fig. 1 ) of the elastic-plastic case. $\left(A_{r r}\right.$ is, however, zero in Eq. (16): the radial strain cannot have a $1 / r$ singularity, as this would imply a logarithmically divergent radial displacement).

This same freedom of the elements to closely duplicate the near tip elastic-plastic field is ruled out from the start in more conventional treatments $[11,12]$ employing "constant strain" elements, which necessarily assign a single set of displacement components to the tip and allow no possibility of a strain singularity.

Two modifications of the element design, not employed in the present study, can be noted here. First, when strain singularities of the form $r^{-1 /(1+N)}$ are appropriate $(N>0)$, as for power law hardening in Eq. (8), one may choose forms for displacements in the nearest tip elements which include terms varying as $r^{N /(1+N)}$. Displacements at $r=0$ then no longer vary with $\theta$, and coefficients of such terms become "generalized displacements" replacing actual nodal displacements in the finite element Eqs. (18). Also, the polar coordinate elements will not in general allow simple descriptions of external specimen boundaries. It is, however, apparent from Eq. (14) that these are simply a type of isoparametric [18] element. Any general set of four-sided isoparametric elements can accomplish the same displacement variation at $r=0$, provided a complete side of elements at the crack tip is mapped into a single point so that separate nodal points coincide at the tip. Such a mapping leads to a $1 / r$ strain singularity when different displacements are taken for the coincident nodal points, and hence should also be suitable for the non-hardening case.

\section{Small Scale Yielding Numerical Formulation}

Following Rice $[4,5]$, the small scale yielding elastic-plastic problem is formulated as that of a semi-infinite crack in an infinite body, with boundary conditions that the stress field of the characteristic elastic singularity (Eqs. 1) is approached as $r \rightarrow \infty$. Such solutions represent complete elastic-plastic solutions in the limiting case when the yield zone size is small in comparison 
to characteristic dimensions, and the elastic stress intensity factor governs local yielding. Comparison with available complete solutions (antiplane strain, Barenblatt-Dugdale model, etc.) suggests that such small scale yielding solutions are accurate approximations up to a range 0.5 to 0.7 of net section yield loads [5].

The solution for monotonic loading is then self-similar, for by dimensional considerations the stresses may depend on $r$ only through the combination $\sigma_{0}^{2} r / K^{2}$ where $\sigma_{0}$ is the tensile yield stress and $K$ the stress intensity factor. This self-similarity cannot be exploited in the solution, as no suitable numerical procedure is available for the resulting equations. We therefore follow the conventional technique of building up the solution in linearized increments, but take advantage of the known self-similarity in presenting numerical results.

The semi-infinite crack in an infinite body is replaced by a cut-out cylinder of finite radius $r_{f}$, containing the crack tip as its origin. $\sigma_{r r}$ and $\sigma_{r f}$ from Eqs. (1) are taken as the normal and tangential boundary tractions on the outer radius. The cylindrical cross-section is covered by elements of the type shown in Fig. 2. Only the region $y>0$ need be considered by symmetry, and 25 rays at equal angular intervals of $7.5^{\circ}$ are placed there. 20 concentric circles are drawn which, together with the crack tip, form radial boundaries of the elements. Letting $r_{0}$ be the radius of the innermost circle, the first 19 are placed at $r_{0},(1.5)^{2} r_{0},(2.0)^{2} r_{0}, \ldots,(9.5)^{2} r_{0},(10.0)^{2} r_{0}$, and the last at $r_{f}=120 r_{0}$. The progression in squares is chosen so that equal increments in $K$ will newly yield approximately equal numbers of elements. In all, 480 elements and 525 nodal points or 1050 degrees of freedom are used. Results are obtained only up to a maximum plastic zone extent of approximately $9 r_{0}$, so the outer radius is effectively always at $\infty$.

In addition to the stress boundary conditions on the outer radius, the normal displacement and shear traction on the ray $\theta=0$ are taken as zero by symmetry. Also, the radial displacement $u_{r}\left(r_{f}, 0\right)$ is taken as zero to remove indeterminate rigid body motions. A suggested by the transition from Eqn. (11) to (12), nodal forces are defined in the sence of equivalent virtual work of the actual boundary tractions on the assumed displacement functional form of Eq. (14). Since strains are not constant within elements, the master stiffness matrix is formed by integrating a quadratic expression in strain increments over each element. This is done by dividing each element into 9 (i.e., 3 by 3 ) polar rectangles, of equal $r$ and $\theta$ spacings, evaluating the integrand at the midpoint of each rectangle, and approximating the integral by a sum. One essential simplification is that whenever the current stress state enters the relation between $\dot{\sigma}_{i j}$ and $\dot{\varepsilon}_{i j}$, as for elements in the plastic range, that state is taken as the stresses associated with the area average strain history of the element. It is easy to see from averaging Eq. (16) for $\varepsilon_{i j}$ that the area average values are given by the same expression evaluated at the mid-radius and angle of an element. All subsequently reported stresses within elements are those based on the area average strains.

The elastic solution corresponding to the load which brings area average stresses within a single element to incipient yielding is calculated first. Then the load (i.e., stress intensity factor $K$ ) is successively incremented by $10 \%$ of this value, the linearized elastic-plastic equations being solved in each increment. In all 30 such increments are added. Poisson's ratio is taken as 0.3 .

The computer program has several time-saving features. For example, many elements have identical stiffness matrices in the elastic range, and only one needs to be generated. Thus 20 elastic stiffness matrices are generated (corresponding to the 20 concentric circles) for all the 480 elements used. Moreover, the element stiffness matrices are stored on disk, once they are formed and at each load increment only the stiffness matrices for the elements which are yielding or have previously yielded are reformed. Typically, in the early stage of loading history, less than 10 element stiffness matrices need be reformed at each load increment. On the IBM 360/50, the elastic solution took 6 minutes in C.P.U. time, while each subsequent increment was completed in 3 minutes in C.P.U. time.

The governing Eqs. (13) were solved in each increment through the Gauss-Seidel iterative scheme, employing an over-relaxation factor. Since the choice of the over-relaxation factor is critical in determining the rate of convergence, the program calculates an approximate value of 
the optimal factor from numerical results in the first few interations, as suggested in Ref. [19]. The calculations were done on the IBM S360/50 at Brown University. Additional details are given in the thesis by Levy [20].

\section{Results}

\section{Initial elastic solution}

The elements were chosen to closely duplicate the singularity in the elastic-plastic rather than the elastic case. Nevertheless, creditable results are obtained in the finite element solution for initial elastic loading, which is required to start the elastic-plastic computation. Area average stresses from this solution first meet the yield condition in one of the innermost elements at a stress intensity factor

$$
K_{0}=2.08 \sigma_{0} r \frac{3}{b}
$$

where $r_{0}$ is the radius of the innermost elements. Computing area average stresses from the exact elastic solution of Eqs. (1), one finds $K_{0}=2.06 \sigma_{0} r_{0}^{\frac{1}{2}}$. However, the exact solution shows that yield first occurs in the element between $82.5^{\circ}$ and $90^{\circ}$, whereas the finite element solution first yields the element between $67.5^{\circ}$ and $75^{\circ}$. This is not so significant a discrepancy as it may seem at first sight. For example, in the numerical solution, the $82.5^{\circ}-90^{\circ}$ element is within $2 \%$ of yield when the $67.5^{\circ}-75^{\circ}$ element yields. Also, from area average stresses computed from the exact solution, the $67.5-75^{\circ}$ element is within $3.5 \%$ of yield when the $82.5^{\circ}-90^{\circ}$ element yields.

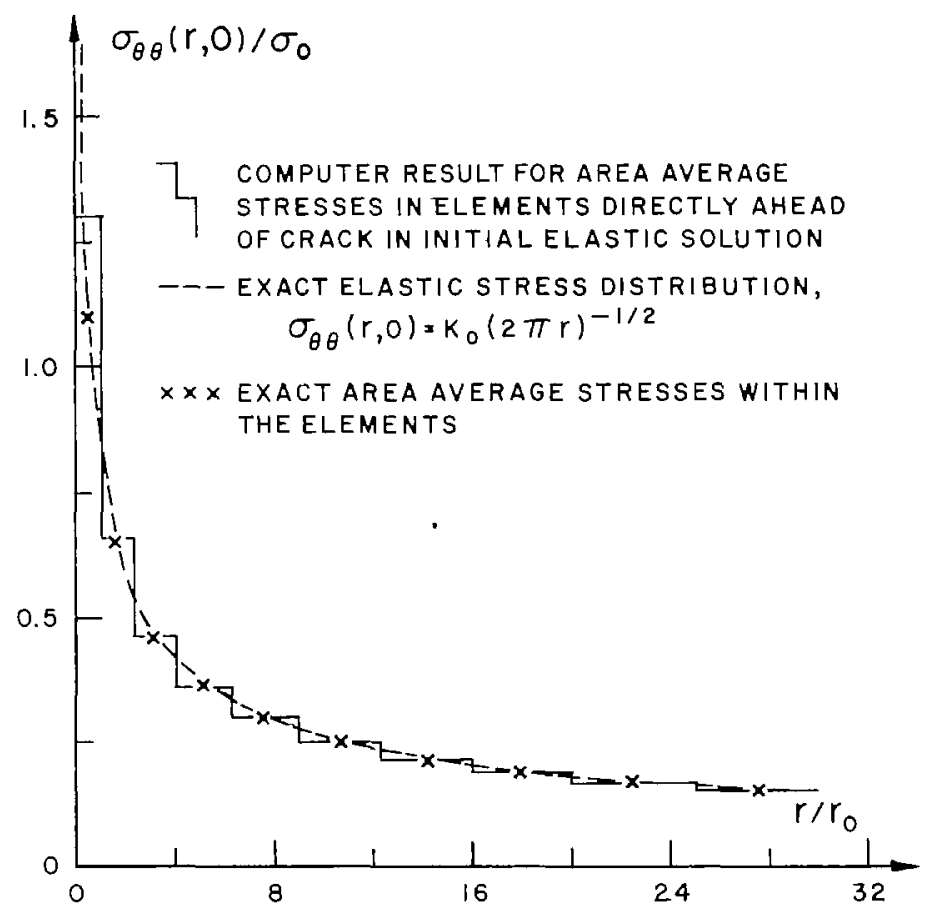

Figure 3. Companson of numencal results for inutial elastic loading with exact elastic stress distribution.

Figure 3 shows, in bar graph form, area average numerical results for $\sigma_{\theta \theta}$ in the elements forming the $7.5^{\circ}$ wedge ahead of the crack tip. These may be compared with the dashed line plot of the exact solution, $\sigma_{\theta \theta}=K_{0}(2 \pi r)^{-\frac{1}{2}}$ for the stress acting across the line ahead of the crack. The more appropriate comparison is with area averages of the exact solution, and these are shown by the crosses placed at the center of each element range. There is a significant discre- 

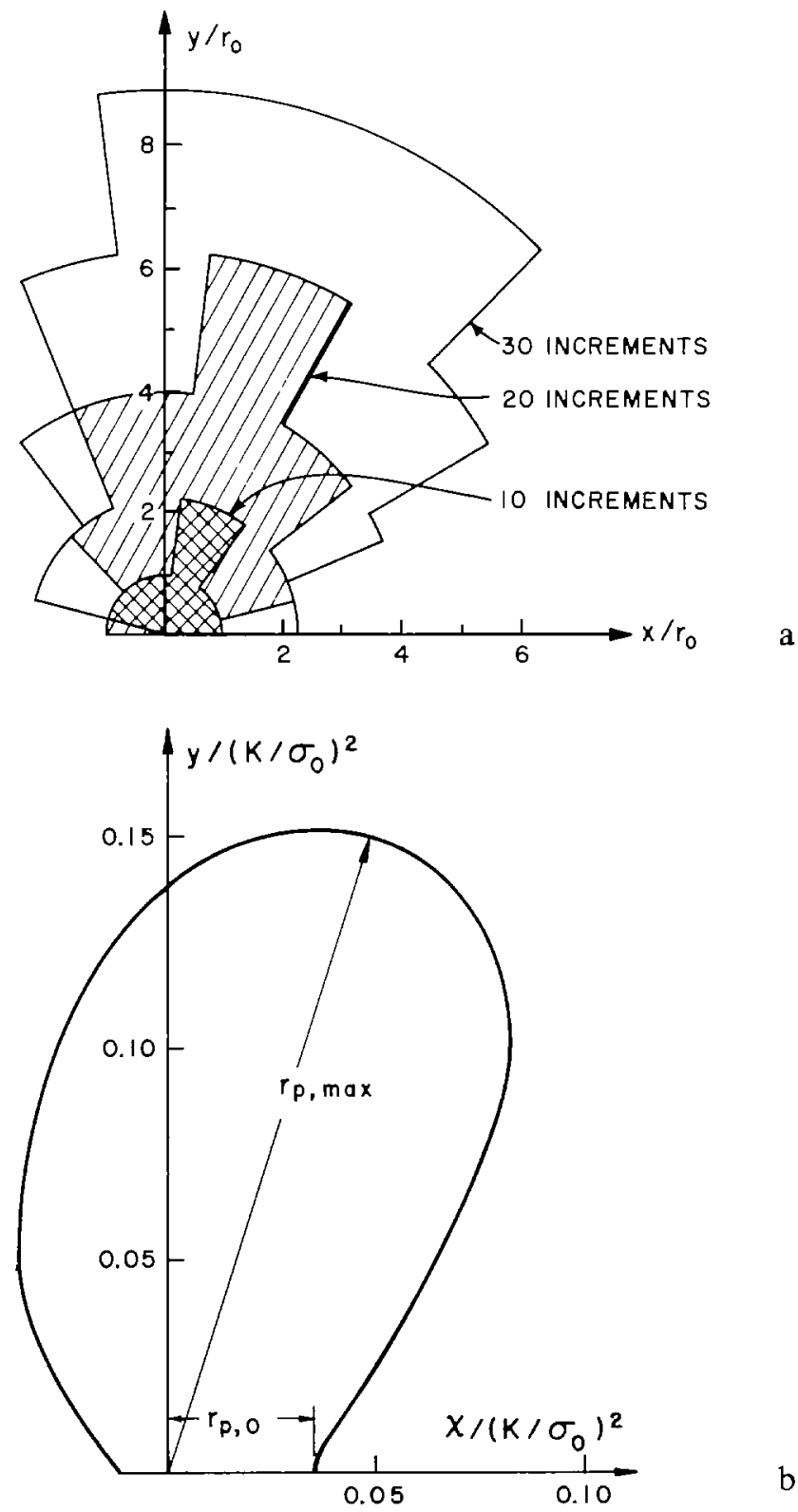

$\mathrm{b}$

Figure 4. Growth of the plastıc zone. (a) Outlines of yielded finite elements after 10,20, and 30 elastic-plastic load increments, with coordinates made dimensionless by radius $r_{0}$ of the innermost elements. (b) Smoothed estimate of elasticplastic boundary, plotted in terms of similarity parameter $\left(K / \sigma_{0}\right)^{2}$ for small scale yielding.

pancy in the element immediately adjacent to the crack tip, with the exact area average about $15 \%$ less than the numerical area average.

Growth of the plastic zone.

Successive linearized elastic-plastic finite element solutions are computed for increments in $K$ of $10 \%$ of $K_{0}$. Figure 4 a shows the outer boundaries of the yielded elements after 10,20 , and 30 of these increments. In Fig. $4 \mathrm{~b}$ we have shown a smoothed estimate of the elastic-plastic boundary, obtained from interpolation and extrapolation of numerical results in the neighborhood of the 30 th increment. Here, advantage has been taken of the known self similarity of the 
solution and $x, y$ distances in the plot are measured in terms of the characteristic length parameter $K^{2} / \sigma_{0}^{2}$. It is seen that the maximum radius to the elastic-plastic boundary occurs at $\theta \approx 70^{\circ}$, the maximum radius and the radius where the elastic-plastic boundary crosses the line ahead of the crack being

$$
r_{p, \max }=0.157 \mathrm{~K}^{2} / \sigma_{0}^{2}, \quad r_{p, 0}=0.036 \mathrm{~K}^{2} / \sigma_{0}^{2} .
$$

Near crack tip elastic-plastic stress state.

Figures $5 \mathrm{a}, \mathrm{b}$, and $\mathrm{c}$ illustrate the success of our finite element design in elucidating the fine details of the near crack tip elastic-plastic field. Taken from numerical results after 30 load

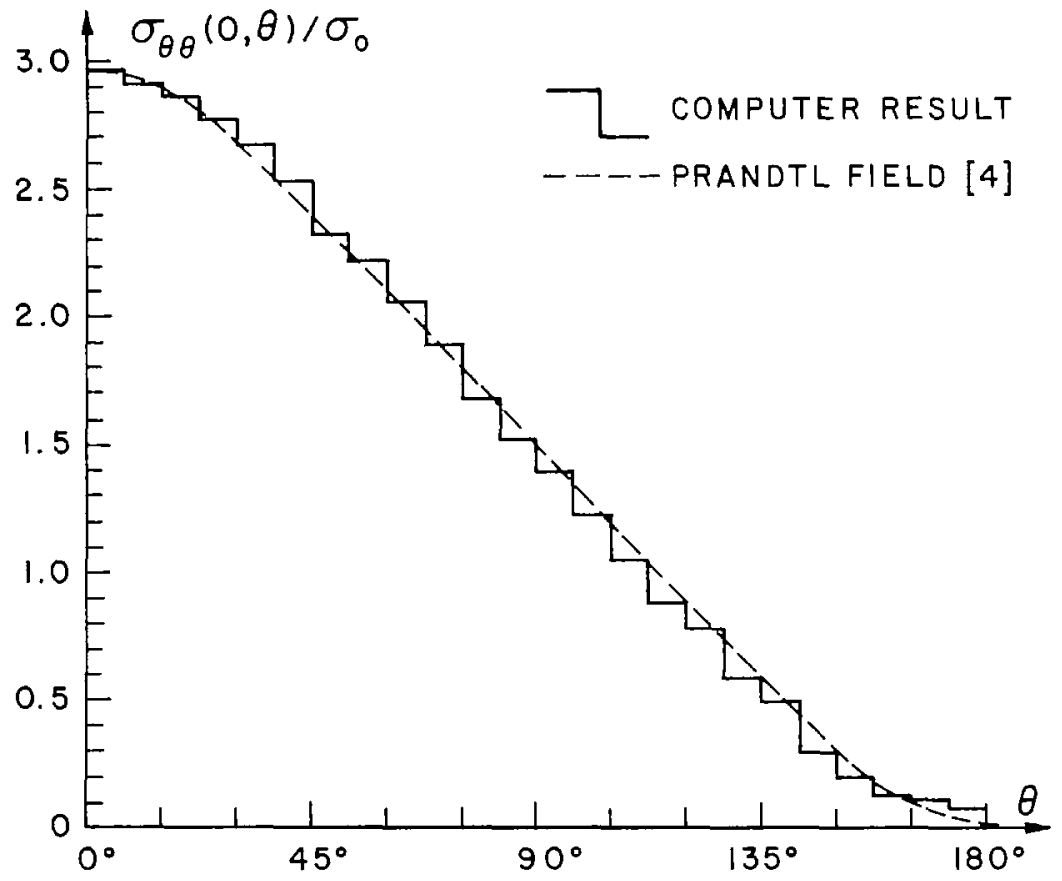

Fig. 5a

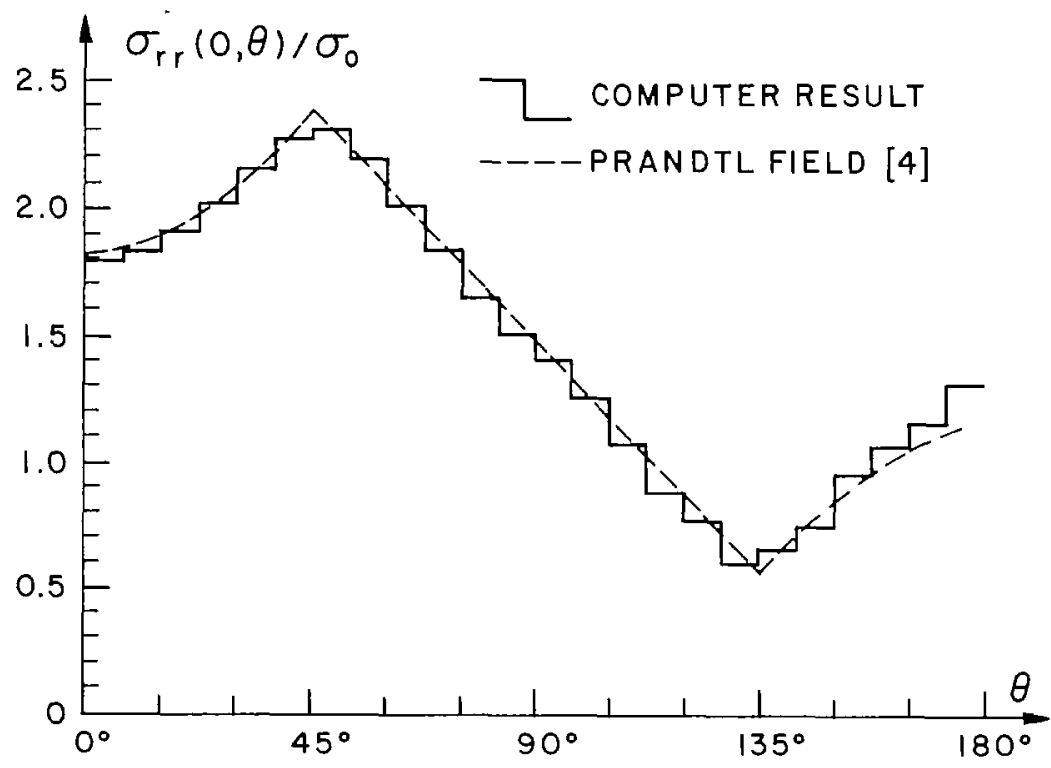

Fig. $5 b$ 


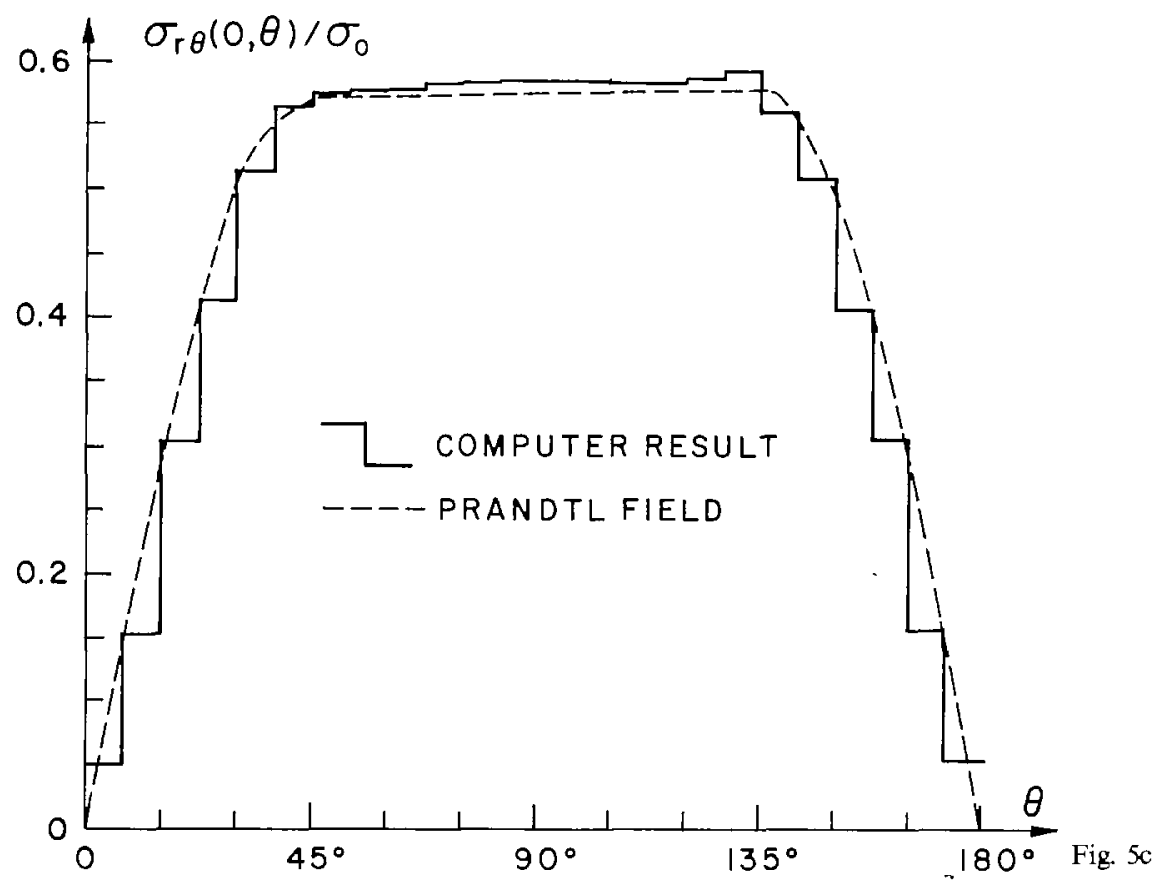

Figure 5. Comparison of numerical results for stresses in innermost elements with exact Prandtl field elastic-plastic stress distribution as in Fig. 1. (a) For $\sigma_{\theta \theta}(0, \theta)$. (b) For $\sigma_{m}(0, \theta)$. (c) For $\sigma_{r \theta}(0, \theta)$.

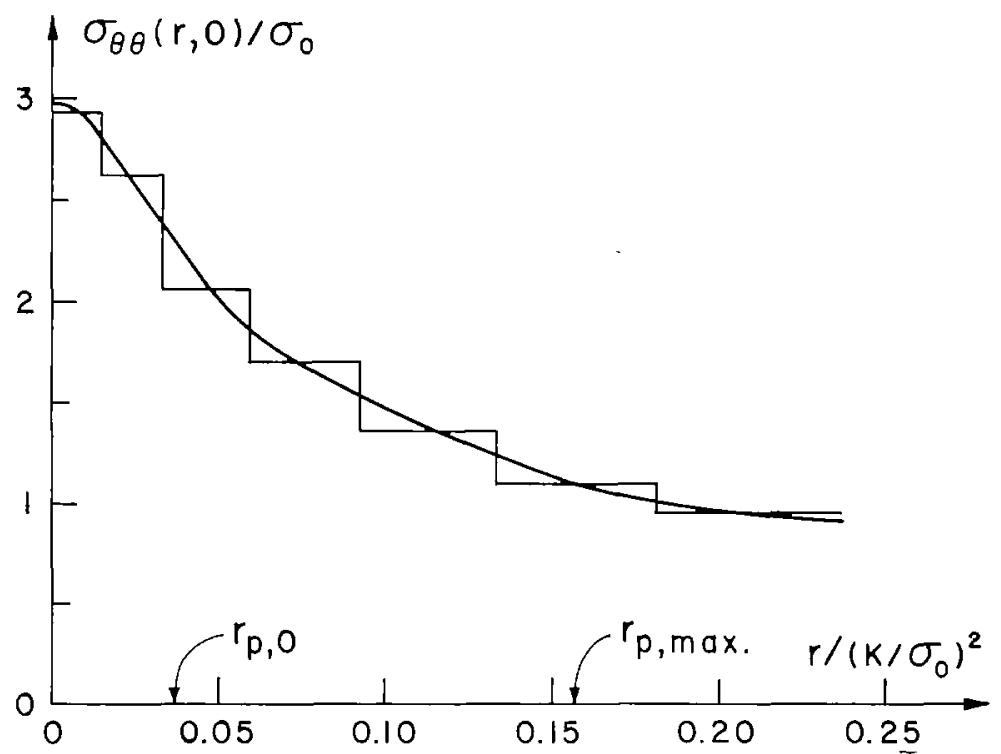

Figure 6. Elastic-plastıc stress distribution ahead of the crack tip, with distance from tup made dimensionless by sim1larity parameter $\left(K / \sigma_{0}\right)^{2}$. Extent $r_{p, 0}$ of plastic zone ahead of crack and distance $r_{p, \text { mex }}$ equal to maximum radius of plastic zone (occurring at $\theta \approx 70^{\circ}$ ) are marked.

increments, these figures show the stresses $\sigma_{\theta \theta}, \sigma_{r r}$ and $\sigma_{r \theta}$ in the 24 innermost finite elements, adjacent to the crack tip. The essentially coincident dashed lines represent the Prandtl stress distribution of Fig. 1, which Rice [4] predicted as the limiting elastic-plastic stress variation with $\theta$ as $r \rightarrow 0$.

The stress distribution directly ahead of the crack tip is shown in Fig. 6, as based on numerical results in the 30th increment. Advantage has again been taken of the self similarity, with distance from the crack tip measured in terms of the dimensionless parameter $r /\left(K / \sigma_{0}\right)^{2}$. For apprecia- 
tion of the size scales, the plastic zone dimensions of Eqs. (18) and Fig. $4 \mathrm{~b}$ are marked on the abscissa. Note that while the theoretical value of $\sigma_{\theta \theta}=3 \sigma_{0}$ is indeed achieved at the tip, the fall-off in stress is very rapid with approximately $2 \sigma_{0}$ resulting where the elastic-plastic boundary cuts the line ahead of the tip, and approximately $\sigma_{0}$ resulting at a distance from the tip equal to the maximum extent of the plastic zone.

Strength of the strain singularity.

The function $R(\theta)$ is introduced in Eq. (4) for describing the strength of the singularity in shear strain within the centered fan directly above the crack tip. This may be computed from the displacements of the coincident nodal points at the crack tip by writing

$$
R(\theta)=\frac{\sqrt{3} E}{(1+v) \sigma_{0}} \lim _{r \rightarrow 0} r \varepsilon_{r \theta}=\frac{\sqrt{3} E}{2(1+v) \sigma_{0}}\left[\frac{\partial u_{r}(0, \theta)}{\partial \theta}-u_{\theta}(0, \theta)\right]
$$

Thus, we report average numerical values for an element lying between $\theta_{1}$ and $\theta_{2}$ by the formula

$$
R\left(\theta_{1} \text { to } \theta_{2}\right)=\frac{\sqrt{3} E}{2(1.3) \sigma_{0}}\left[\frac{u_{r}\left(0, \theta_{2}\right)-u_{r}\left(0, \theta_{1}\right)}{\theta_{2}-\theta_{1}}-\frac{u_{\theta}\left(0, \theta_{2}\right)+u_{\theta}\left(0, \theta_{1}\right)}{2}\right] .
$$

Figure 7 shows the numerical results for $R(\theta)$ over an angular range corresponding to the fan. Here, advantage has been taken of the self similarity and effects of inaccuracies in the initial elastic-plastic transition of the innermost elements has been filtered out (see below) by taking differences between numerical results in the 30th and 15 th increments. The maximum value of $R(\theta)$ is

$$
R_{\max }=0.155\left(K / \sigma_{0}\right)^{2},
$$

and it occurs in the element between $82.5^{\circ}$ and $90^{\circ}$. Also, the function falls off essentially to zero at the $45^{\circ}$ and $135^{\circ}$ boundaries of the centered fan. The two additional plots in Fig. 7 are from deformation plasticity analyses based on the path independent $J$ integral, and we discuss the discrepancies later. One curve is from Rice's approximation [4], in which the functional form of $R(\theta)$ was assumed apart from a multiplicative constant determined by the integral. The other curve is from the non-hardening limit $N \rightarrow 0$ of the Cherepanov-Hutchinson-Rice-Rosengren

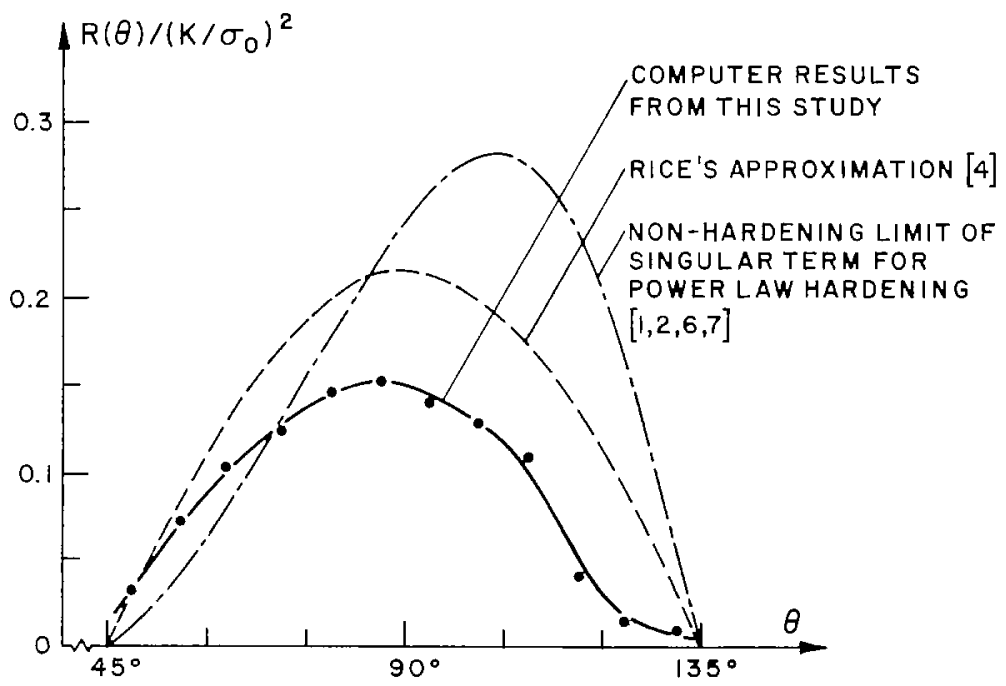

Figure 7. Dimensionless plot of the strength $R(\theta)$ of the inverse with distance strain singularity in the centered fan region directly above the crack tip. Numerical results based on incremental plastıcity theory differ significantly from two earlier approximations based on deformation theory. 
power law hardening singularity which was, however, not given in their original papers, but rather later in Ref. [7].

Crack opening displacement.

The total separation distance between upper and lower crack surfaces at the tip is given by $\delta_{\mathrm{t}}=-2 u_{\theta}(0, \pi)$. The increase of this quantity with stress intensity factor is shown in Fig. 8, and

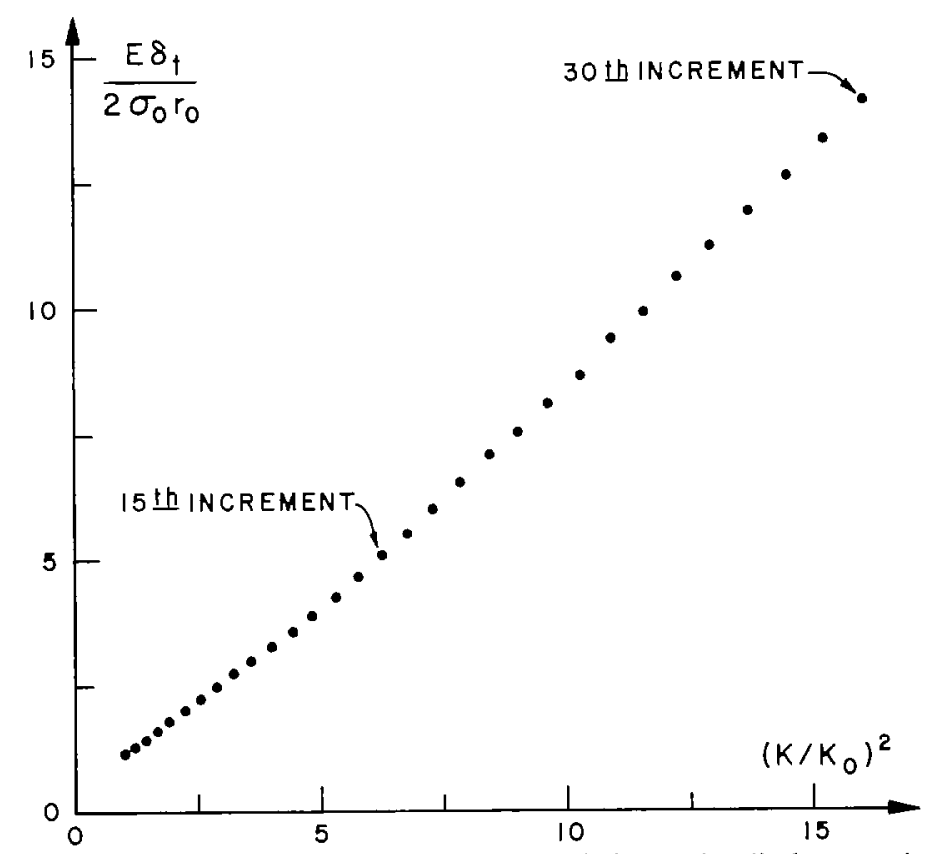

Figure 8. Points show numerical results for crack tip opening displacement in the successive elastic-plastic increments. The linear vanation with $K^{2}$, as required theoretically from self-similarity of exact solution, results after sufficient number of finite elements have yielded.

this variation is typical of all crack tip displacements in the numerical results. We interpret the deviation at low $K$ from the essentially linear variation with $K^{2}$, as required by dimensional analysis, to be due to extraneous effects in the initial yielding of elements before the plastic region is fully developed. Based on a straight line difference between results in the 15 th and 30 th increments, the crack tip opening displacement is

$$
\delta_{\mathrm{t}}=0.425 \frac{K^{2}}{E \sigma_{0}} .
$$

For comparison, determination of $\delta_{t}$ through Eq. (5), involving integration of numerical results for $R(\theta)$, leads to a factor of approximately $0.41-0.42$. Also, the two deformation plasticity analyses based on the $J$ integral lead to a factor of 0.613 in Rice's [4] approximation, and 0.717 in the non-hardening limit of the power law hardening singularity [7].

\section{Discussion}

One surprising result, illustrated in Fig. 7, is the significant difference in the strength $R(\theta)$ of the strain singularity for the present incremental plasticity solution, as compared to the two approximations based on deformation plasticity analyses. Since both deformation theory approximations for $R(\theta)$ lead, through the integral of Eq. (6), to the value of $J$ given in Eq. (7), it is clear that $R(\theta)$ from the incremental solution must lead to a smaller value for the integral. In fact, integration of numerical results reads to a value of the integral in Eq. (6) which is approxi- 
mately $\frac{3}{4}$ of the value in Eq. (7). If the present results are indeed an accurate representation of the incremental solution, this would suggest that, in an averaged sense, the strength of the strain singularity in the incremental solution is about $\frac{3}{4}$ of that in the deformation solution.

These results are not entirely unexpected when it is recalled that proof of path independence of the $J$ integral, which implies equality between the integral of Eq. (6) and the value for $J$ in Eq. (7), relies strongly on path independence of the stress-strain relation [4]. This latter path independence is not a feature of a proper incremental formulation of plastic stress-strain relations, and thus the $J$ integral theory is rigorously applicable in an incremental material only if proportional plastic straining results. It is easy to see that proportional plastic straining does not result in the present problem. For if Poisson's ratio is less than $\frac{1}{2}$ and if the sum of in-plane normal stresses is non-zero, the flow rule for a Mises material results in a non-zero plastic strain increment in the $z$ direction at initial yield. However, as plastic strains become large compared to elastic strains, as near the crack tip singularity, the constraint $\varepsilon_{z z}=0$ forces further plastic strain increments to be essentially in the plane of straining with near zero components in the $z$ direction. In particular, the $1 / r$ singular term reflects only in-plane plastic strain increments, with zero increments in the $z$ direction.

It is also of interest to note that the distribution of $R(\theta)$ from the non-hardening limit of the power law hardening singularity does not, according to our present results, give the correct distribution in the complete elastic-plastic solution. Indeed, there is no real reason that it should, although one might have expected this result from comparison with the anti-plane strain case [6], for which corresponding function is correct with small scale, but not large scale, yielding.

Rice and Johnson [7] have shown the elastic-plastic plane strain solution, based on the small geometry change assumption for a sharp crack, can be used to set boundary conditions for an approximate analysis of the large but highly localized deformations associated with crack tip blunting. Consideration of such large geometry change effects appears to be essential for understanding the physical mechanisms of plane strain fracture. Unfortunately, the bulk of their study was completed before the present solution became available, and they based their treatment of fracture under small scale yielding conditions on Rice's [4] approximate analysis for $R(\theta)$ and the associated crack tip velocity field. Their paper does, however, present a comparison of that velocity field with the results of the computer solution, and the relative closeness of the two suggests that their results would be little modified, except that the present Eq. (22), for relating $\delta_{t}$ to the stress intensity factor, should be adopted in lieu of the equation they employed.

\section{Acknowledgment}

Early phases of the work reported here were supported by the Advanced Research Projects Agency under contract SD-86, with the main part of the study supported by the National Aeronautics and Space Administration under grant NGL-40-002-080.

\section{REFERENCES}

[1] G. P. Cherepanov, Crack Propagation in Continuous Media, Appl. Math. Mech (PMM), 31 (1967) 476.

[2] J. W. Hutchinson, Singular Behavior at the End of a Tensile Crack in a Hardening Material, J. Mech. Phys. Solids, 16 (1968) 13.

[3] J. W. Hutchinson, Plastic Stress and Strain Fields at a Crack Tip, J. Mech. Phys. Solids, 16(1968) 337.

[4] J. R. Rice, A Path Independent Integral and the Approximate Analysis of Strain Concentration by Notches and Cracks, J. Appl. Mech., 35 (1968) 379.

[5] J. R Rice, Mathematical Analysis in the Mechanics of Fracture, Chp. 3 of Fracture, An Advanced Treatise, Vol. II (H. Liebowitz, ed.), Academic Press, New York, 1968.

[6] J. R. Rice and G. F. Rosengren, Plane Strain Deformation Near a Crack Tip in a Power Law Hardening Material, J. Mech. Phys. Solids, 16 (1968) 1.

[7] J. R. Rice and M. A. Johnson, The Role of Large Crack Tip Geometry Changes in Plane Strain Fracture, to appear in Inelastic Behavior of Solids (M. F. Kanninen et al, eds.), McGraw-H1ll, New York. (Proc. of Sept. 1969 Battelle Colloquium on Inelastic Behavior of Solıds.) 
[8] M. L. Williams, On the Stress Distribution at the Base of a Stationary Crack, J. Appl. Mech., 24 (1957) 109.

[9] P. V. Marcal and I. P. King, Elastic-Plastic Analysis of Two-Dimensional Stress Systems by the Finite Element Method, Int. J. Mechanical Sciences, 9 (1967) 143.

[10] A. Mendelson, Plasticity: Theory and Application The Macmillan Company, New York, 1968.

[11] J. L. Swedlow, A. H. Yang and M. L. Williams, Elasto-Plastic Stresses and Strains in a Cracked Plate, in Proc. of the First Int. Conference on Fracture, Sendai, 1965 (T. Yokobori et al., eds), vol I. p. 259, Japanese Society for Strength and Fracture of Materials, Tokyo, 1966.

[12] J. L. Swedlow, Elasto-Plastic Cracked Plates in Plane Strain, Int. J. Fracture Mech, 5 (1969) 33.

[13] I. S. Tuba, A Method of Elastic-Plastic Plane Stress and Strain Analysis, J. of Strain Analysis, 1 (1966) 115.

[14] P. Hilton and J. W. Hutchinson, Plastic Intensity Factors for Cracked Plates, Report SM-34, Harvard University, May 1969, to appear in Engr, Fracture Mech

[15] P. C. Paris and G. C. Sih, Stress Analysis of Cracks, in Fracture Toughness Testing and Its Applications, STP-381, p. 30, Amer. Soc. Testing and Materials, Philadelphia, 1965.

[16] D. C. Drucker and J. R. Rice, Plastic Deformation in Brittle and Ductile Fracture, Engr. Fracture Mechanics, to appear $1969-70$

[17] D. C. Drucker, Variational Principles in the Mathematical Theory of Plasticity, Proc. Symp. Appl. Math, Volume 8, Calculus of Variations and its Applications, p. 7, 1958.

[18] O. C. Zienkiewicz and Y. K. Chung. The Finite Element Method in Structural and Continuum Mechanics, McGrawHill, 1967.

[19] D. Young, The Numerical Solution of Elliptic and Parabolic Partial Differential Equations, in Survey of Numerical Analysis (J. Todd, ed.), McGraw-Hill, 1962.

[20] N. Levy, Application of the Finite Element Method to Large Scale Elastic-Plastic Problems of Fracture Mechanics, $P h$ D. Thesis, Brown University, July 1969.

\section{RÉSUME}

L'article présente une solution par éléments finus en conditions élastoplastiques, au problème de l'écoulement plastıque de faible étendue qui se produit au voisinage d'une fissure dans un matériau non vieıllissant en conditions d'état plan de déformation.

On met l'accent sur le mode de traçage des éléments finıs le meux susceptible de reproduire avec exactitude, en solution numérique, la configuration détaillée des champs de contrainte et de déformation à l'extrémité de la fissure; référence est faite, à cet égard, à des études analytiques précédentes sur les singularités de l'extrémité d'une fissure.

Des résultats numériques ont été obtenus pour: le lieu de la frontière élastoplastique, la distribution angulaire de l'intensité de la singularité des déformations, le déplacement d'ouverture de l'extrémité de la fissure, la distribution des contraintes en avant de la fissure, et la variation angulaire des contraintes à l'extrémité de la fissure.

Ces derniers résultats, qui fournissent la distribution limite des contraintes lorsque $r$ tend vers zéro, confirment l'existence d'un champ de Prandtl, ainsi que Rice l'avait prédit.

\section{ZUSAMMENFASSUNG}

Der Bericht gibt eine Lösung durch endliche Elemente unter elastoplastischen Bedingungen, für das Problem des plastischen Fliessens über kurze Strecken, welches in der Umgebung eines einem planen Spannungszustand unterworfenen Risses in nichtalterndem Material auftritt.

Die Auslegung der endlichen Elemente, welche es am besten ermöglicht eine genaue Reproduzierbarkeit (bei numerischer Lōsung) der detaillierten Struktur der Spannungs- und Verformungsfelder in der Umgebung der RiBspitze zu erreichen, unter Bezug auf frühere analytische Untersuchungen über die Singularitat einer RiBspitze, wird besonders herausgestrichen.

Numerische Ergebnisse werden gegeben für:

die Lage der elastoplastischen Grenze, die angulàre Verteilung der Intensität der Verformungssingularität, die Verschıbung der Rißspitzenöıfnung, die Spannungsvertellung vor dem Rısse, sowie für die anguläre Veränderung der Spannungen an der Spitze.

Letztere Ergebnisse liefern die Grenzvertellung dẹr Spannungen wenn $r \rightarrow 0$ und bestätigen die Voraussagen von Rice über die Existenz eines Prandt-Feldes.

Int. Journ. of Fracture Mech., 7 (1971) 143-156 